\title{
Direct Determination of Ascorbic Acid in a Grapefruit: Paving the Way for In Vivo Spectroelectrochemistry
}

\author{
Jesus Garoz-Ruiz, Aranzazu Heras,* and Alvaro Colina* \\ Department of Chemistry, Universidad de Burgos, Pza. Misael Bañuelos s/n, E-09001 Burgos, Spain
}

\begin{abstract}
The research field of real samples is pretty much complicated than the study of other systems. However, the inherent advantages of UV-vis absorption spectroelectrochemistry should overcome some difficulties related to direct measurements in complex matrices. For this reason, a singular spectroelectrochemistry device has been fabricated and validated. The novel cell is based on single-walled carbon nanotubes which are filtered and subsequently press-transferred on a polyethylene terephthalate support using a stencil with a custom design. With this new methodology, working, counter, and reference electrodes are completely flat on the surface where two optical fibers are fixed in a long optical path length configuration. In order to demonstrate the usefulness of this device and the power of spectroelectrochemistry techniques to solve problems of the current world, this device is used to quantitatively detect the concentration of ascorbic acid in a complex matrix such as a fruit, directly, without any previous sample pretreatment. The ease to fabricate the device, the advantages related to its use, and the excellent results obtained not only with univariate but also with multivariate analysis, shed more light on the analysis of samples as they occur in nature. According to the particular features of this cell, to the best of our knowledge this is the first spectroelectrochemical sensor that can be inserted directly in a biological matrix, laying the groundwork to perform in vivo measurements in a near future.
\end{abstract}

Teamwork is a decisive factor in the current world to solve important problems in all working environments. Increasingly, companies require to their employees broad powers in terms of dialogue and teamwork skills. In parallel, the development of analytical techniques based on the successful marriage between techniques of different nature allows researchers to obtain fascinating results.

Spectroelectrochemistry ${ }^{1,2}$ is a "teamwork technique" that joins the best of electrochemistry and spectroscopy together. This hybrid technique studies simultaneously not only the electrochemical but also the spectroscopic evolution of an electron-transfer process. Therefore, spectroelectrochemistry, by definition, allows us to obtain information with at least two signals of different nature at the same time, an uncommon but very powerful feature. As can be inferred, this multiresponse technique has been applied in a large number of fields, including, for example, electron transfer processes, ${ }^{3}$ reaction mechanisms, ${ }^{4}$ electrocatalysis, ${ }^{5}$ conducting polymers, ${ }^{6}$ solar cells, ${ }^{7}$ memory devices, ${ }^{8}$ supercapacitors, ${ }^{9}$ synthesis of nanoparticles, ${ }^{10}$ carbon nanomaterials, ${ }^{11}$ characterization of metal complexes, $^{12}$ liquid/liquid interfaces, ${ }^{13,14}$ electrochromic materials, ${ }^{15}$ and the study and determination of compounds of biological interest. ${ }^{16-19}$ It is noteworthy that, at present, the technical developments allow us to perform spectroelectrochemistry measurements with much chemical information for the quantification of analytes and for the comprehension of their reaction mechanisms. ${ }^{16,18,20-22}$

Nanotechnology is playing a crucial role during these last years, improving the quality of life in many different fields. In this sense, the importance of carbon nanomaterials, in particular carbon nanotubes, ${ }^{23,24}$ is evident. This fact is due to the unique and exceptional properties of carbon nanotubes, highlighting mechanical, thermal, optical, electronic, and catalytic properties, $^{25,26}$ that make them a highly promising carbon allotrope with a wide range of applications. ${ }^{27,28}$ Particularly, the use of carbon nanotubes in different branches of medicine, $^{29-32}$ specially sensors, ${ }^{33-35}$ is increasing steadily in recent years, with particular emphasis on biocompatibility. ${ }^{36}$ In general, electrodes based on carbon nanotubes are extensively used for electroanalytical applications, and, definitively, in the electrochemical detection of biomolecules. ${ }^{18,37-39}$ In fact, single-walled carbon nanotubes (SWCNTs) networks are very effective for trace level measurements which have proved inaccessible to other electrode materials. ${ }^{40}$ According to these 
excellent electrochemical properties of SWCNTs, an interconnected network of this carbon nanomaterial acts as a fantastic electrode for electrochemistry and spectroelectrochemistry. ${ }^{41,42}$

As is well known, there are interesting works about electrochemistry in complex samples, being even possible to perform in vivo electrochemistry. ${ }^{43-47}$ Nevertheless, although spectroelectrochemistry offers inherent advantages and provides more information about physicochemical processes than electrochemistry, the promising field of the study of complex matrices and living or real samples by spectroelectrochemistry ${ }^{48-50}$ is still taking its first steps. Actually, to the best of our knowledge, and apart from few very interesting devices, ${ }^{51-53}$ there is a lack of UV-vis absorption spectroelectrochemistry devices that allow us to perform direct measurements in complex matrices, probably due to the intrinsic difficulties to develop devices able to carry out such experiments. The main difficulties in complex sample detection are the presence of interfering species, the absence of the in situ character, the high cost of the equipment, the long analysis times, or the difficulty of the experimental devices. Aiming to solve these problems and to shed more light in these scientific areas, a new spectroelectrochemistry device is proposed in this work, totally movable and capable of being inserted in in vivo samples. As demonstrated below, this device overcomes the drawbacks mentioned before.

With this objective, we rely on two developments performed in our group that have aroused great interest in the research community. The first one is the development of several methodologies to fabricate SWCNT networks by filtration to be transferred on different supports for spectroelectrochemistry applications, ${ }^{18,41,54}$ among others. The second one is the use of bare optical fibers without cladding material to conduct the light beams in parallel and bidimensional spectroelectrochemistry. ${ }^{18,54,55}$ It is worth noting, with respect to this last point, the successful use of optical fibers in biochemical applications. $^{56}$

By combining all the advantages demonstrated in these works, a novel device with the three electrodes completely flat in the support and the optical fibers attached thereto has been successfully developed. This UV-vis absorption spectroelectrochemistry device is used to fulfill the main goal of this work, which is the direct determination of compounds of biological interest in a complex matrix, without any previous sample pretreatment. As a proof of concept, a grapefruit was chosen as target to quantify directly its ascorbic acid (AA) concentration.

Undoubtedly, the easy-to-use device presented in this work provides independent and complementary information and brings together the best features of both electrochemistry and spectroscopy to contribute to the use of spectroelectrochemistry in real environments.

\section{EXPERIMENTAL SECTION}

Reagents and Materials. SWCNTs (Sigma-Aldrich), 1,2dichloroethane (DCE, 99.8\% for HPLC, Acros Organics), polytetrafluoroethylene (PTFE) membranes (filter pore size $0.1 \mu \mathrm{m}$, JVWP01300, Millipore Omnipore), polyethylene terephthalate (PET, $175 \mu \mathrm{m}$ thick, HiFi Industrial Film), silver conductive paint (Electrolube), $\mathrm{HCl}$ (35\%, VWR), and trans- parent nail polish (Essence) were used to fabricate the electrodes. Two bare optical fibers $(100 \mu \mathrm{m}$ in diameter, Ocean Optics) were needed to fabricate the spectroelectrochemistry device. Ferrocenemethanol (FcMeOH, 97\%, Acros Organics), $\mathrm{KCl}$ (Acros Organics), AA (L(+)-ascorbic acid, Acros Organics), $\mathrm{LiClO}_{4}$ (Panreac), and 2,6-dichloroindophenol sodium salt hydrate (DCIP, Sigma-Aldrich) were used to prepare the solutions.

All reagents were used as received without further purification. All chemicals were of analytical grade. Aqueous solutions were freshly prepared using ultrapure water (18.2 $\mathrm{M} \Omega$ $\mathrm{cm}$ resistivity at $25^{\circ} \mathrm{C}$, Milli-Q Direct 8, Millipore).

Instrumentation. Spectroelectrochemistry setup includes a potentiostat/galvanostat (PGSTAT302N, Metrohm Autolab), a halogen-deuterium light source (AvaLight-DH-S-BAL, Avantes), and a spectrometer (QE65000 198-1006 nm, Ocean Optics). A tip-sonicator (CY-500, Optic ivymen System) was also used to disperse the SWCNTs in DCE. Stencils were made of polymethyl methacrylate (PMMA, Maniplastic) using a high-precision $\mathrm{CO}_{2}$ laser cutting machine. A laboratory hydraulic press (SpectroPress, Chemplex Industries, Inc.) was also used to transfer the SWCNT film, obtained by filtration, on the PET substrate.

Safety Considerations. All handling and processing were performed carefully, particularly when DCE was used.

Transference of SWCNTs Using a Stencil. The transference of carbon nanotubes films of different shapes on many supports is required for several applications. Herein, we present a methodology based on filtering a SWCNT dispersion and press-transferring the SWCNT film formed to a PET sheet using a stencil with the desired shape.

The first step to achieve a good SWCNT transference is to obtain a homogeneous dispersion of $0.5 \mathrm{mg}$ of SWCNTs in $100 \mathrm{~mL}$ of DCE. The procedure followed in this work has already been described elsewhere. ${ }^{54}$ Briefly, $0.5 \mathrm{mg}$ of SWCNTs were weighed and sonicated in increasing volumes of DCE applying different sonication power levels. $10 \mathrm{~mL}$ of the final dispersion were sonicated before preparing the transfers to avoid SWCNT agglomeration and to ensure a good homogeneity. Subsequently, $0.8 \mathrm{~mL}$ of the SWCNT dispersion were filtered under vacuum through a PTFE filter, resulting in a homogeneous film of SWCNTs on the membrane surface. By filtering $0.8 \mathrm{~mL}$ of the dispersion through a membrane area of $0.785 \mathrm{~cm}^{2}$, the amount of SWCNTs that remained in the membrane was enough to form an interconnected network of SWCNTs with highlighted conductivity. ${ }^{41}$ Next, filter was dried at room temperature for only five minutes because longer times imply wrong transferences. Afterward, the filter was placed between the stencil and the PET support, which was previously washed with water and dried, taking care that the polymeric substrate did not acquire static electricity because it decreases the transference quality. Figure S1 of the Supporting Information shows an illustration about the experimental setup used to fabricate the SWCNT press-transfers with a stencil. Finally, the filter was carefully detached using tweezers. As a result, SWCNTs were transferred on the PET support according to the chosen shape of the stencil.

Fabrication of the Electrochemical Device. The preparation process of the electrodes is quite simple. Electrical con- 
tacts were made with silver conductive paint. One of the small transfers was also covered with this material aiming to constitute the reference electrode (RE). Next, silver conductive paint was dried at $75{ }^{\circ} \mathrm{C}$ for 45 minutes. A drop of $\mathrm{HCl}$ in water, 1:10 dilution, was added to the silver RE to form a $\mathrm{Ag} / \mathrm{AgCl}$ RE. Finally, the electrical contacts were electrically isolated with nail polish which was dried at room temperature. This insulator is also used to control the working electrode (WE) area. The excellent reproducibility of electrodes based on the press-transference of carbon nanomaterials was demonstrated in one of our previous works. ${ }^{42}$ Therefore, a three-electrode cell with a WE and a counter electrode (CE) of SWCNTs and a Ag/AgCl RE has been fabricated.

Fabrication of the Spectroelectrochemistry Device. Two bare optical fibers of $100 \mu \mathrm{m}$ in diameter were easily and perfectly aligned, opposite one another, and fixed with nail polish to the PET support, allowing the parallel light beam to pass only through the first $100 \mu \mathrm{m}$ of solution layer adjacent to the SWCNT WE. The optical path length is defined as the distance between the ends of the two bare optical fibers, which can be easily controlled with the use of a piece of the desired length, helping to study the electron transfer of compounds with very different molar absorption coefficients. For the sake of clarity, Figure 1 shows an illustration to scale of the final spectroelectrochemistry device. In addition, a photograph of the spectroelectrochemistry device with the different steps of its fabrication can be observed in Figure S2 of the Supporting Information.

Experimental Setup for UV-vis Absorption Spectroelectrochemistry Measurements. The ease to perform spectroelectrochemistry measurements is one of the key points of this device. As is shown in Figure 1, the electrical contacts are connected to the potentiostat, one of the optical fibers to the UV-vis light source and the other one to the spectrometer. The transmitted parallel light beam collects the spectral changes that occur between the optical fibers and that are related to the processes that take place only in the first $100 \mu \mathrm{m}$ of the electrode-solution interface, being the electrochemical signal simultaneously recorded in a single experiment. The potentiostat and the spectrometer are properly synchronized using a trigger that guarantees the simultaneous character of all spectroelectrochemistry experiments.

The detection mechanism of the device toward the target molecules is based on the principles of spectroelectrochemistry in long optical path length

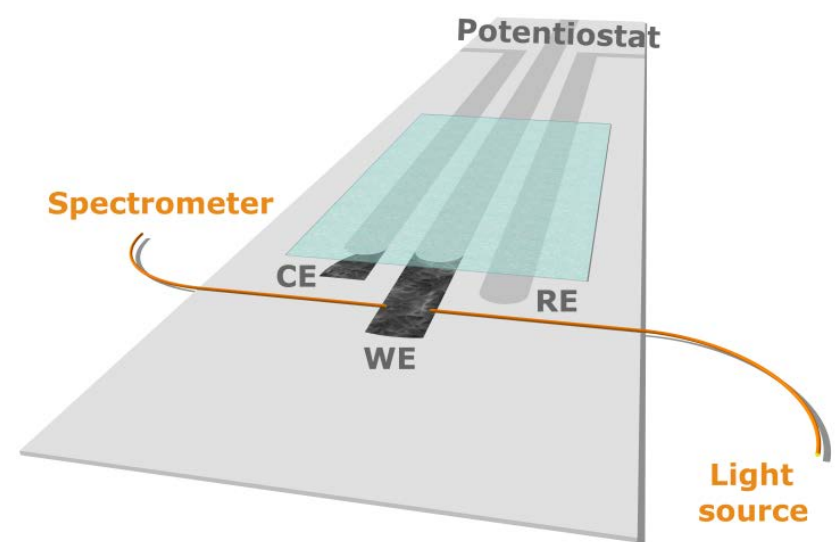

Figure 1. Illustration of the spectroelectrochemistry device. The detection mechanism of the device toward the target molecules is described in the main text.

arrangement. The analyte is oxidized or reduced in the surface of the WE, where is electrochemically detected, and, simultaneously, the oxidized or reduced analyte is spectroscopically detected when it diffuses from the WE surface to the bulk solution. In our case, as the diameter of the bare optical fibers, which are fixed to the support, is $100 \mu \mathrm{m}$, the oxidized or reduced analyte is spectroscopically detected in the first 100 $\mu \mathrm{m}$ of the diffusion layer of the solution adjacent to the WE surface.

Inherent advantages associated with long optical path length spectroelectrochemistry devices such as the one developed herein, should be highlighted instead of common normal configuration devices: (i) a higher sensitivity and, thus, lower limits of detection; (ii) the use of optically transparent electrodes is not required; and (iii) the light beam only samples the first micrometers of the solution adjacent to the electrode surface, so the spectral changes recorded during the electrochemical reaction are only related to the electrode-solution interface.

Several great advantages related to the new transfer methodology and the spectroelectrochemistry device presented in this work should be mentioned. Filtering SWCNT dispersions without surfactants and removing the filter without chemicals allow us to obtain homogeneous, reproducible, and clean SWCNT transferences. Furthermore, the use of stencils with a customized design guarantees a great versatility about the number, shapes, and sizes of the transferences, and, in this case, of the electrodes. Any stencil can be designed; hence, any three-electrode spectroelectrochemistry cell can be fabricated. Moreover, the different transferences made at the same time with the stencil serve to remove the typical homemade $\mathrm{Ag} / \mathrm{AgCl} / \mathrm{KCl} 3 \mathrm{M} \mathrm{RE}$ and the platinum or gold wire CE used in previous works, ${ }^{18}$ making it possible to perform spectroelectrochemistry experiments in a simpler way. In addition, the three electrodes are completely flat on the surface of the PET support where the optical fibers are strongly attached, allowing a good reproducibility and an easy mobility of the whole device. One of the main advantages of this spectroelectrochemistry device and this experimental setup is the high versatility. A lot of parameters, such as the optical path length or the integration time, can be selected by the researcher for each problem to be solved. For example, the control of the optical path length allows us to improve the sensitivity and the capability of detection of the spectroelectrochemical sensor. As demonstrated below, this device enables us to perform spectroelectrochemistry measurements in complex matrices. In addition, this work serves as a starting point for future in vitro experiments, being a promising approach to in vivo spectroelectrochemistry.

\section{RESULTS AND DISCUSSION}

Validation of the Spectroelectrochemistry Device. FcMeOH has been used to validate the device. This compound undergoes a reversible electrochemical process of one electron in a simple, well-defined, and diffusion-controlled way, being 
widely used as a probe in electrochemistry and recently in spectroelectrochemistry. $^{54}$

Several spectroelectrochemistry experiments of $5 \times 10^{-4} \mathrm{M}$ FcMeOH in $0.1 \mathrm{M} \mathrm{KCl}$ between $-0.20 \mathrm{~V}$ and $+0.50 \mathrm{~V}$ at different potential scan rates are represented in Figure 2 and Figure S3 of the Supporting Information. The optical path length was set at $1.45 \mathrm{~mm}$ and a full spectrum was taken every $250 \mathrm{~ms}$, for which the halogen-deuterium light source was employed because the spectral changes related to $\mathrm{FcMeOH}$ take place in the UV (deuterium) and in the visible (halogen) region. $^{54}$ A $100 \mu \mathrm{L}$ solution drop, which was changed after every measurement, was placed in the spectroelectrochemistry device covering the three electrodes and the ends of the optical fibers, allowing us to work in a semi-infinite diffusion regime.

Figure 2a shows the 3D plot of the spectra evolution with time/potential recorded during a cyclic voltammetry performed at a potential scan rate of $0.010 \mathrm{~V} \mathrm{~s}^{-1}$. The corresponding cyclic voltammogram (blue line of Figure 2b) shows an anodic peak at $+0.185 \mathrm{~V}$ and a reduction peak at $+0.099 \mathrm{~V}$. As has been previously reported, ${ }^{54}$ the three absorption bands observed in Figure 2a at 260, 282, and $630 \mathrm{~nm}$ evolve according to the potentiodynamic experiment, and are attributed to the electrogeneration of the oxidized form of the redox couple, $\mathrm{FcMeOH}^{+}$, taking into account that the reduced form, FcMeOH (initial solution), was taken as reference spectrum.

In order to compare both the electrochemical and spectroscopic responses, the cyclic voltammogram at $0.010 \mathrm{~V} \mathrm{~s}^{-1}$ is overlapped with the corresponding voltabsorptogram at 282 $\mathrm{nm}$ (blue and green lines of Figure 2b, respectively). The great agreement between the two signals assures that the light beam passes through the first $100 \mu \mathrm{m}$ of the solution layer closest to the SWCNT WE and that both the potentiostat and spectrometer are properly synchronized.

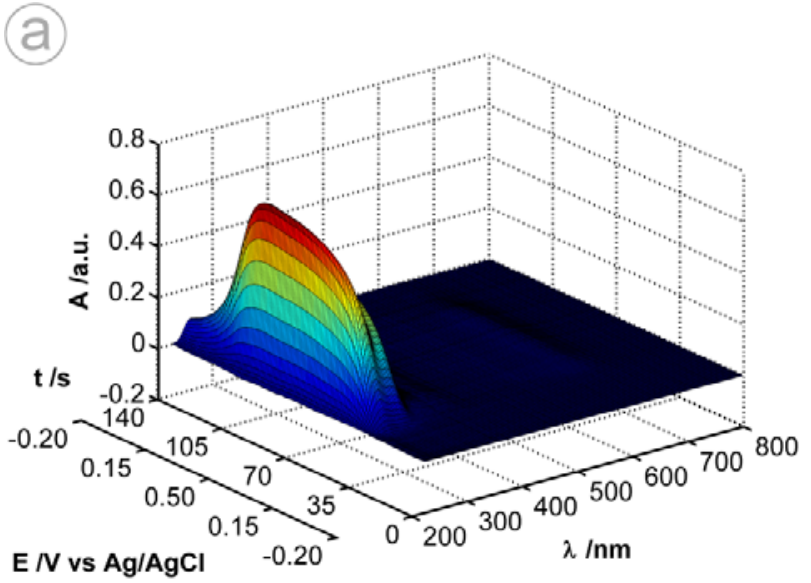

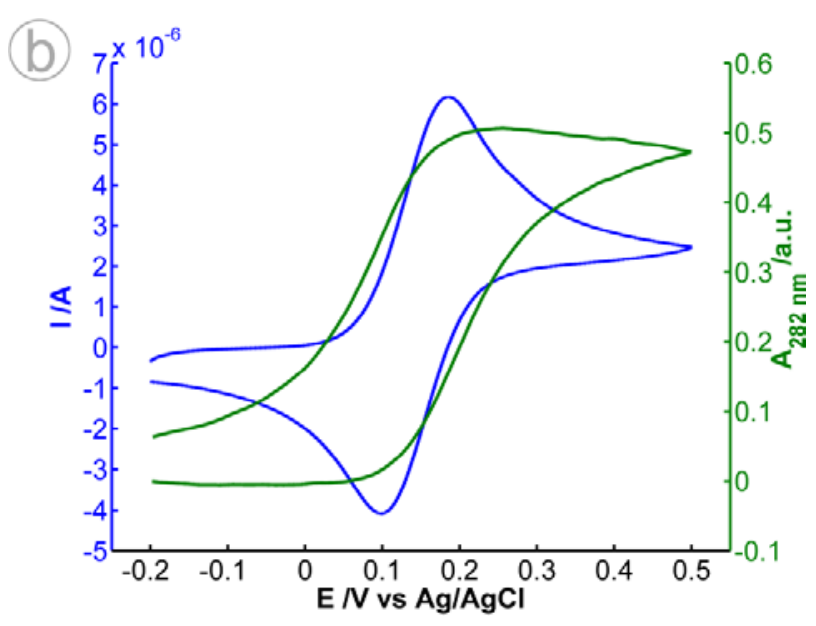

Figure 2. Spectroelectrochemistry experiments of $5 \times 10^{-4} \mathrm{M}$ $\mathrm{FcMeOH}$ in $0.1 \mathrm{M} \mathrm{KCl}$ between $-0.20 \mathrm{~V}$ and $+0.50 \mathrm{~V}$ at a potential scan rate of $0.010 \mathrm{~V} \mathrm{~s}^{-1}$. (a) 3D plot of the spectra evolution with time/potential recorded during the cyclic voltammetry (cyclic voltammogram plotted in Figure 2b). (b) Comparison between the cyclic voltammogram and the voltabsorptogram at $282 \mathrm{~nm}$.

The electrochemical reaction produces a concentration gradient that is observed in the voltabsorptograms at $282 \mathrm{~nm}$ at different potential scan rates shown in Figure S3 of the Supporting Information, where are briefly described along with the two different behaviors that can be observed taking into account the potential scan rate. In addition, an experiment of $0.1 \mathrm{M} \mathrm{KCl}$ at $0.010 \mathrm{~V} \mathrm{~s}^{-1}$ was carried out at the end of all the measurements to demonstrate that there are no spectral changes with potential in a blank solution. Therefore, parallel measurements include information about the global quantity of $\mathrm{FcMeOH}^{+}$present in the electrode-solution interface, its diffusion, its spatial distribution, and its position with respect to the electrode surface.

All these observations are entirely consistent with previous results. ${ }^{54}$ The validation of this novel spectroelectrochemistry device demonstrates its excellent performance. Next, it is used to quantify directly the amount of AA present in a complex matrix.

Determination of AA in a Complex Matrix. AA, as other vitamins, is an essential biomolecule that must be ingested through diet. Deficiency of AA is related to scurvy, ${ }^{57}$ thus, its determination, for example by electrochemical methods, ${ }^{58}$ is highlighted. It should be noted that AA has been scarcely studied by UV-vis spectroelectrochemistry, ${ }^{51,59,60}$ while the quantification of AA by this in situ technique has been performed in an indirect way by using a mediator. ${ }^{61}$

The need to simplify and improve the existing methodologies to carry out experiments in a suitable manner for real samples and the powerful features associated with spectroelectrochemistry techniques have led us to perform the work presented herein. Taking all these facts into account, and once that the experimental UV-vis spectroelectrochemistry device has been validated, the quantitative determination of AA directly in a grapefruit is carried out without any sample pretreatment.

Potentiostatic spectroelectrochemistry measurements of different concentrations of AA between $1 \times 10^{-4} \mathrm{M}$ and $3.5 \times$ 
$10^{-3} \mathrm{M}$ in $0.1 \mathrm{M} \mathrm{LiClO}_{4}$, applying a constant potential of $+0.90 \mathrm{~V}$ during $60 \mathrm{~s}$, were performed to construct a calibration curve, assuring that the electrochemical oxidation of AA was taking place independently of the matrix studied, as will be indicated below. Seven cycles in a cyclic voltammetry experiment in $0.1 \mathrm{M} \mathrm{LiClO}_{4}\left(\mathrm{E}_{\mathrm{i}}=-0.50 \mathrm{~V}, \mathrm{E}_{\mathrm{v} 1}=+1.40 \mathrm{~V}\right.$, and $\mathrm{E}_{\mathrm{v} 2}=$ $-1.40 \mathrm{~V}$ ) at $0.050 \mathrm{~V} \mathrm{~s}^{-1}$ were performed prior to the calibration curve to condition the SWCNT WE. Spectral changes related to AA only occur in the UV spectral range, especially around $252 \mathrm{~nm}$, so that only the deuterium light source was used. As the AA concentration in the grapefruit is presumably high, the optical path length was shortened to $0.45 \mathrm{~mm}$, allowing to reduce the integration time to $110 \mathrm{~ms}$ to obtain more data, compared to the parameters of $1.45 \mathrm{~mm}$ and $250 \mathrm{~ms}$ of the previous validation section. A $200 \mu \mathrm{L}$ solution drop was placed in the spectroelectrochemistry device and the initial AA solution of each experiment was taken as reference spectrum. This set of calibration samples was employed to evaluate the capability of the spectroelectrochemistry device to quantify AA. An example of the results obtained using a solution $1 \times$ $10^{-3} \mathrm{M}$ AA in $0.1 \mathrm{M} \mathrm{LiClO}_{4}$ and applying a constant potential of $+0.90 \mathrm{~V}$ during $60 \mathrm{~s}$ is shown in Figure 3 .

The chronoamperogram shown in Figure 3a is associated with the electrochemical reaction corresponding to the oxidation of AA to dehydro-L-ascorbic acid (DHA) whose carbonyl groups undergo hydration yielding an electroinactive product. $^{58,62}$ The overall reaction can be summarized in the following simplified mechanism:

$$
\begin{aligned}
& \mathrm{AA} \rightleftarrows \mathrm{DHA}+2 \mathrm{H}^{+}+2 \mathrm{e}^{-} \\
& \mathrm{DHA}+\mathrm{H}_{2} \mathrm{O} \rightarrow \mathrm{C}
\end{aligned}
$$

This process involves not only the transfer of two electrons but also two protons, acidifying the solution where the electrochemical reaction takes place.

The inset of Figure 3a shows the 3D plot of the spectra evolution with time obtained during the amperometric experiment. It displays the decrease of the absorbance in the UV region, concomitantly with the electrochemical signal, that is related to the consumption of AA, according to the reaction mechanism. Therefore, the oxidation process of AA to DHA is observed both in the chronoamperogram and, simultaneously, in the UV region, by the decrease of the absorbance.

The evolution of the absorbance at $252 \mathrm{~nm}$ is represented in Figure 3b. This wavelength was selected because $252 \mathrm{~nm}$ is an isosbestic point where ascorbate monoanion and AA have the same molar absorption coefficient. ${ }^{63,64}$ As can be seen, the decrease of the absorbance at $252 \mathrm{~nm}$ from the beginning of the experiment confirms the oxidation of AA to DHA and its diffusion from the SWCNT WE surface to the bulk solution when a potential of $+0.90 \mathrm{~V}$ is applied. Once again, the good agreement between electrochemical and spectroscopic signals should be highlighted, making it possible to corroborate the electrochemical behavior of AA taking into account the spectral response.
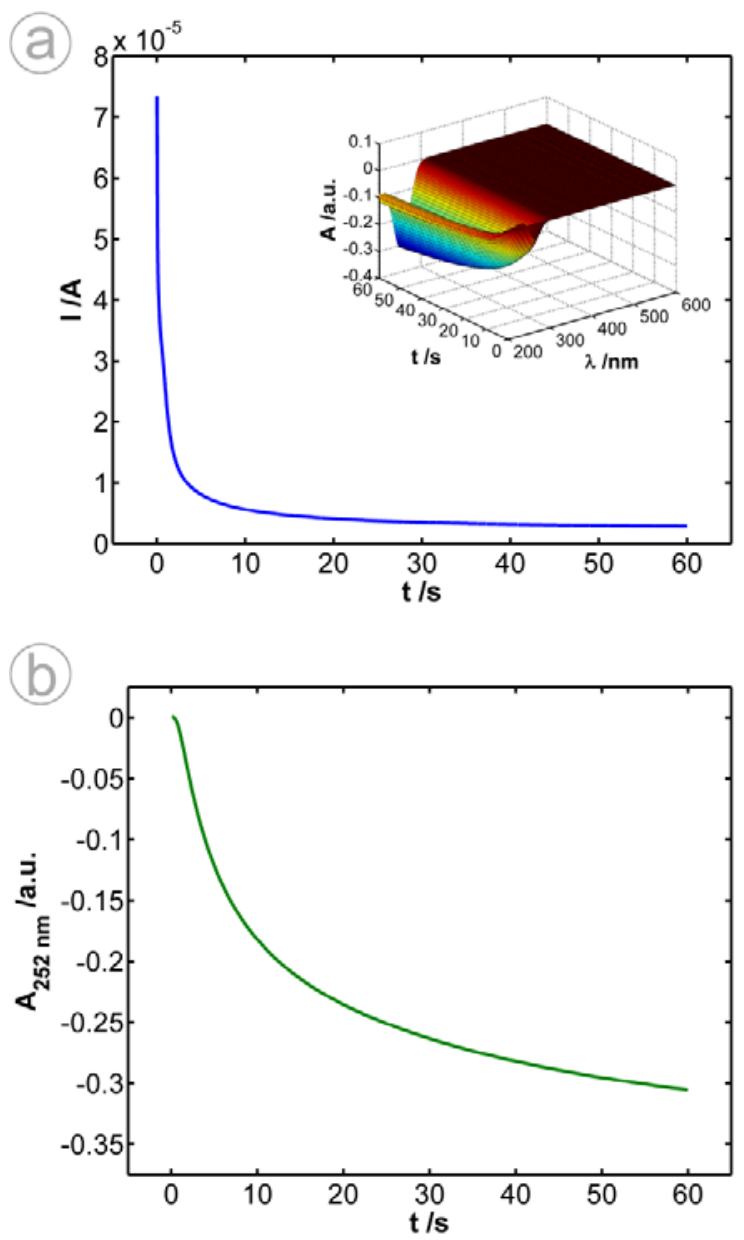

Figure 3. Spectroelectrochemistry experiment of $1 \times 10^{-3} \mathrm{M}$ AA in $0.1 \mathrm{M} \mathrm{LiClO}_{4}$ applying a constant potential of $+0.90 \mathrm{~V}$ during 60 s. (a) Chronoamperogram obtained. Inset: 3D spectra evolution with time recorded during the electrochemical signal. (b) Evolution of the absorbance at $252 \mathrm{~nm}$.

When a set of calibration samples of AA, such as the one outlined above, is analyzed, the higher current intensities and the higher absorbance changes at $252 \mathrm{~nm}$ are related to the increase in the AA concentration. In view of the electrochemical and optical signals shown in Figure 3, short times are associated with a better sensitivity for an electrochemical calibration while long times are related to a better sensitivity for a spectroscopic calibration. Then, $25 \mathrm{~s}$ was selected as an optimal time to provide good results, aiming to sample both signals at the same time.

Therefore, two univariate calibration curves in the $1 \times 10^{-4}$ $\mathrm{M}-3.5 \times 10^{-3} \mathrm{M}$ concentration range were obtained using an ordinary least squares (OLS) regression model and taking (i) the current intensity at $25 \mathrm{~s}$ and (ii) the absorbance at $252 \mathrm{~nm}$ at the same time, for the electrochemical and the spectroscopic signals, respectively, versus AA concentration. Both electrochemical and spectroscopic univariate calibration curves (panels a and b of Figure S4 of the Supporting Information, respectively) were tested to evaluate their prediction capability. For this purpose, the prediction of the concentration of a test sample of $1.6 \times 10^{-3} \mathrm{M} \mathrm{AA}$ in $0.1 \mathrm{M} \mathrm{LiClO}_{4}$ was performed under the same experimental conditions described above. The re- 
gression parameters of these calibration curves along with the predicted concentrations obtained with both of them for the test sample of AA are displayed in Figure S4 of the Supporting Information and in the first two rows of Table 1 . As can be observed, good relationships between the two variables and AA concentration were obtained. In addition, both electrochemical and spectroscopic calibration curves correctly estimate the concentration of the test sample of AA, whose value is included in the corresponding confidence intervals for the prediction, being the relative standard deviation (\%RSD) for the estimations lower than $4 \%$. All these good figures of merit demonstrate the suitability of both electrochemical and spectroscopic univariate calibration curves for the quantitative analysis of AA by spectroelectrochemistry. Detection limits were 250 and $100 \mu \mathrm{M}$ for the electrochemical and spectroscopic calibration curves, respectively. Nevertheless, detection limits of this method are lower, 7 and $4 \mu \mathrm{M}$, respectively. However, as shown below, the AA concentration in the grapefruit is relatively high and the calibration curves were constructed accordingly.

After demonstrating that both electrochemical and spectroscopic methods provide valid calibration curves for the determination of AA independently of each other, the predicted concentrations with both of them are compared to corroborate that spectroelectrochemistry is an autovalidated analytical method. This autovalidated character is related to the two independent responses obtained simultaneously for each sample, which is very useful for analytical purposes. By plotting the predicted concentrations obtained with the electrochemical calibration curve versus the predicted concentrations calculated with the spectroscopic calibration curve, the regression parameters were $1.00 \pm 0.07$ for the slope and $[0.05 \pm 1.5] \times$ $10^{-4}$ for the intercept $\left(\mathrm{R}^{2}=0.995, \mathrm{~S}_{\mathrm{yx}}=9.5 \times 10^{-5}\right)$. Consequently, with the slope being equal to unity and the intercept equal to zero, both electrochemical and spectroscopic signals determine AA without distinction, proving that UV-vis absorption spectroelectrochemistry is an autovalidated method for quantitative analysis. Thus, the trueness of this analytical method has been demonstrated according to IUPAC suggestions that indicate that results obtained with a method should be validated with a second and independent one.

Finally, the quantification of AA in a complex matrix such as a grapefruit was performed without any previous pretreatment of the sample. An incision was made in the equatorial region of the fruit and the spectroelectrochemistry device was placed inside. Before all measurements, experiments using cyclic voltammetry were performed in the grapefruit, allowing us to conclude that $+0.90 \mathrm{~V}$ is a potential high enough to make sure that the electrochemical oxidation of AA takes place in the grapefruit. Figure 4 displays a photograph of the experimental setup with the spectroelectrochemistry cell placed inside the grapefruit, not only to show this new methodology but also to give an idea about the great novelty, ease, robustness, and the analytical possibilities opened up by this spectroelectrochemistry device in comparison with traditional spectroelectrochemistry experiments. Figure S5 of the Supporting Information shows the spectroelectrochemistry measurements in the grapefruit, under the same experimental potentiostatic conditions as before. In this figure, the oxidation of AA is easily observed in both electrochemical and spectroscopic responses, exhibiting a behavior very similar to that indicated in Figure 3. As can be observed in the first two rows of Table 1 , values of $[1.99 \pm 0.14] \times 10^{-3} \mathrm{M}$ and $[2.06 \pm 0.11] \times 10^{-3} \mathrm{M}$ are obtained for the concentration of AA in the grapefruit, using the electrochemical and spectroscopic univariate calibration curves, respectively. These two values demonstrate the excellent results achieved.

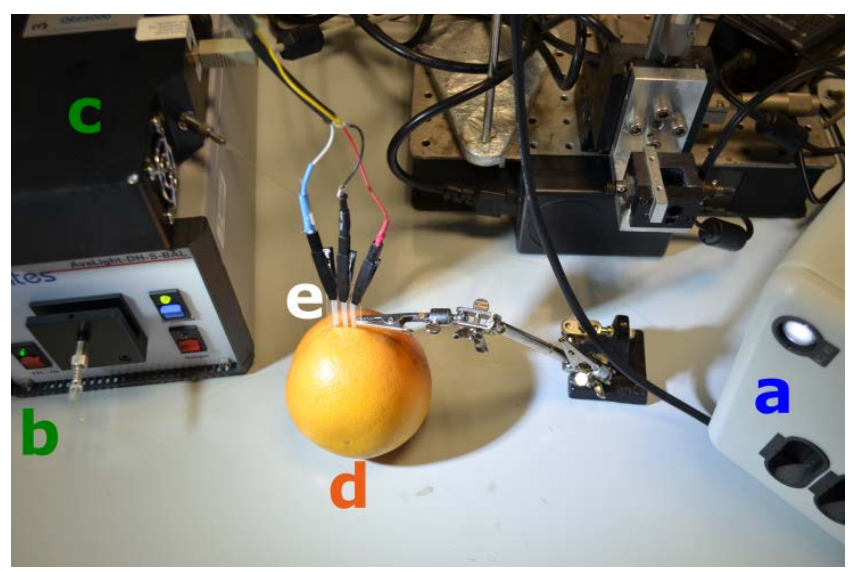

Figure 4. Device placed inside the grapefruit to perform a spectroelectrochemistry measurement. Setup only requires the (a) potentiostat, (b) light source, (c) spectrometer, (d) grapefruit, and (e) spectroelectrochemistry device.

Furthermore, in order to triple check these two values, autovalidated in itself, measurements based on an AOAC official method of titration of AA with DCIP ${ }^{65}$ were carried out immediately after performing the spectroelectrochemistry measurement, obtaining a value of $[2.08 \pm 0.06] \times 10^{-3} \mathrm{M}$ for the concentration of AA in the grapefruit juice. This last result verifies the reliability of the results obtained by UV-vis absorption spectroelectrochemistry for the determination of the AA concentration inside the grapefruit without performing any previous sample pretreatment. In order to prove the innovation related to the spectroelectrochemistry method, two main advantages should be mentioned: (i) spectroelectrochemistry provides two simultaneous and autovalidated results and (ii) a titration is impossible to be carried out directly in a grapefruit.

Specificity and anti-interference properties are inherent to spectroelectrochemistry experiments due to the impossibility of finding two compounds that exhibit exactly the same electrochemical behavior and identical spectroscopic properties. Excellent results in terms of specificity and anti-interference have been achieved using the spectroelectrochemical sensor to determine the AA concentration directly in a grapefruit, as demonstrated by the two values obtained by spectroelectrochemistry and the result obtained with the AOAC method. Being aware of the great variety of compounds present in a fruit sample and its complexity, the spectroelectrochemistry method has proven to be a powerful tool to quantify the amount of an analyte avoiding interferences. Actually, the 3D plot of the spectra evolution with time recorded during the spectroelectrochemistry experiment in the grapefruit (inset of panel a of Figure S5 of the Supporting Information) shows a slight decrease of the absorbance around $325 \mathrm{~nm}$. This wavelength does not interfere at all with the absorption at $252 \mathrm{~nm}$ taken for the spectroscopic univariate calibration curve of AA, but, nevertheless, this situation could have happened. It should 
be noted that much more information can be extracted from spectroelectrochemistry experiments because the electrochemical signal is recorded during $60 \mathrm{~s}$ and a full spectrum between 198 and $1006 \mathrm{~nm}$ is registered with high resolution every 110 ms. For these reasons, partial least squares (PLS) multivariate regression supplies valuable information, improving the reliability of the univariate determination of AA. Three different PLS regression models were built, selecting one latent variable, to obtain three different multivariate calibration curves. On the one hand, current intensities from 25 to 50 s (1251 electrochemical times) were selected versus AA concentration for the electrochemical calibration. On the other hand, two PLS regression analysis were performed for the spectroscopic calibration selecting (i) absorbances in the spectral range between 200 and $350 \mathrm{~nm}$ at $25 \mathrm{~s}$ (187 wavelengths) and (ii) absorbances at $252 \mathrm{~nm}$ from 25 to $50 \mathrm{~s}$ (228 spectral times), versus AA concentration. The regression parameters of these multivariate calibration curves, as well as the predicted con- centrations of AA in the test sample and in the grapefruit obtained with them, are shown in the last three rows of Table 1. The autovalidated character of spectroelectrochemistry is also present in these PLS models. These multivariate models, constructed for quantitative purposes, are more useful than the univariate models to solve complex analytical problems.

\section{CONCLUSIONS AND FUTURE PERSPECTIVES}

A new UV-vis absorption spectroelectrochemistry device based on SWCNTs and bare optical fibers in parallel arrangement has been developed. To do this, a versatile, inexpensive, and quick methodology to transfer SWCNT films on a PET support using customized stencils is proposed. In order to record the spectral changes, two bare optical fibers fixed to the PET substrate sample the solution closest to the SWCNT WE. This easy-to-use device, characterized by its reduced size and the small sample

Table 1. Regression parameters obtained for the determination by spectroelectrochemistry of AA in the concentration range of $1 \times 10^{-4} \mathrm{M}-3.5 \times 10^{-3} \mathrm{M}$ and concentration estimated from univariate (OLS) and multivariate (PLS) calibration curves for a test sample of $1.6 \times 10^{-3} \mathrm{MAA}$ in $0.1 \mathrm{M} \mathrm{LiClO}_{4}$ and for a grapefruit as real sample.

\begin{tabular}{|c|c|c|c|c|c|c|}
\hline \multirow{2}{*}{\multicolumn{2}{|c|}{ Analysis Method }} & \multirow{3}{*}{$\frac{\mathbf{R}^{2}}{0.998}$} & \multirow{3}{*}{$\frac{\mathrm{S}_{\mathrm{yx}}}{2.1 \times 10^{-7}}$} & \multirow{3}{*}{$\begin{array}{c}\text { RMSEP } \\
-\end{array}$} & \multirow{3}{*}{$\frac{\mathbf{C}_{\text {test }}=\mathbf{1 . 6} \times \mathbf{1 0}^{-3} \mathbf{M}}{\mathbf{C I}(\mathbf{M})}$} & \multirow{3}{*}{$\begin{array}{c}\text { Grapefruit } \\
\text { CI (M) } \\
{[1.99 \pm 0.14] \times 10^{-3}}\end{array}$} \\
\hline & & & & & & \\
\hline OLS & $I_{25 \mathrm{~s}}$ & & & & & \\
\hline OLS & $\mathbf{A}_{252 \mathrm{~nm}, 25 \mathrm{~s}}$ & 0.999 & $1.1 \times 10^{-2}$ & - & {$[1.65 \pm 0.11] \times 10^{-3}$} & {$[2.06 \pm 0.11] \times 10^{-3}$} \\
\hline PLS & $\mathbf{I}_{25-50 \mathrm{~s}}$ & 0.998 & - & $4.7 \times 10^{-5}$ & {$[1.61 \pm 0.14] \times 10^{-3}$} & {$[1.92 \pm 0.14] \times 10^{-3}$} \\
\hline PLS & $A_{200-350 n m, 25 s}$ & 0.999 & - & $3.5 \times 10^{-5}$ & {$[1.65 \pm 0.11] \times 10^{-3}$} & {$[2.04 \pm 0.11] \times 10^{-3}$} \\
\hline PLS & $A_{252 \mathrm{~nm}, 25-50 \mathrm{~s}}$ & 0.999 & - & $3.6 \times 10^{-5}$ & {$[1.67 \pm 0.11] \times 10^{-3}$} & {$[2.19 \pm 0.11] \times 10^{-3}$} \\
\hline AOAC $^{65}$ & Titration & & & & & {$[2.08 \pm 0.06] \times 10^{-3}$} \\
\hline
\end{tabular}

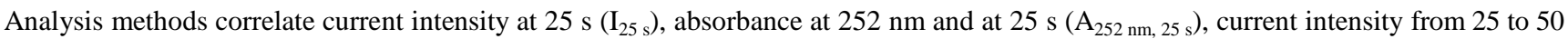

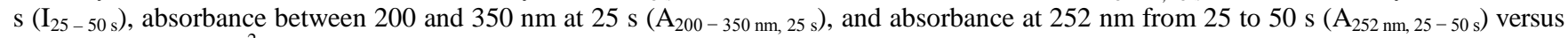
AA concentration. $\mathrm{R}^{2}$, coefficient of determination; $\mathrm{S}_{\mathrm{yx}}$, residual standard deviation; RMSEP, root mean squared error of prediction; $\mathrm{C}_{\text {test }}$, concentration of the test sample of AA; CI, confidence interval.

volume required, allows us to obtain simultaneously electrochemical and spectroscopic information. After being validated with $\mathrm{FcMeOH}$, the reaction mechanism of AA was studied. In order to assess the potential of this movable spectroelectrochemistry device, the determination of AA in a grapefruit has been performed without any pretreatment of the sample, directly placing the device inside this complex matrix. Quantification of AA has been carried out in solution and inside the grapefruit, using both the electrochemical and the spectral signals and making use of the autovalidated character of this hybrid technique. Therefore, our new long optical path length device has solved many problems to bring spectroelectrochemistry to real life.

Based on these results, we are working on new projects related to UV-vis absorption spectroelectrochemistry for the near future: (i) the miniaturization of the device; (ii) the use of PARAFAC to resolve complex mixtures of neurotransmitters and compounds of biological interest with overlapping electrochemical and spectroscopic signals; (iii) the study of other analytes in aqueous solution and in real samples such as tis- sues and biological fluids; and (iv) the development of a novel thin-layer cell.

In summary, the new SWCNT transfer method and the multiple advantages of this device open new perspectives for spectroelectrochemistry. Promising results with complex matrices have been achieved using univariate and multivariate statistical tools, enabling researchers from different disciplines to use spectroelectrochemistry. This work definitely represents a significant advance in this field, paving the way for in vivo spectroelectrochemistry experiments. Not long ago, all this was unthinkable. Now, all qualitative and quantitative information obtained by spectroelectrochemistry demonstrates that this technique is fundamental to address new challenges.

\section{ASSOCIATED CONTENT}

\section{Supporting Information}

Supporting Information Available: 
Experimental SWCNT press-transfers setup, photograph of the spectroelectrochemistry device, voltabsorptograms at $282 \mathrm{~nm}$ of FcMeOH, univariate calibration curves of AA, and spectroelectrochemistry experiments in the grapefruit (PDF).

This material is available free of charge via the Internet at http://pubs.acs.org.

\section{AUTHOR INFORMATION}

\section{Corresponding Authors}

*E-mail: maheras@ubu.es

*E-mail: acolina@ubu.es

Tel: +34 9472588 17. Fax: +34947258831.

\section{ORCID}

Jesus Garoz-Ruiz: 0000-0002-5775-4247

Aranzazu Heras: 0000-0002-5068-2164

Alvaro Colina: 0000-0003-0339-356X

\section{Author Contributions}

The manuscript was written through contributions of all authors. All authors have given approval to the final version of the manuscript.

Notes

The authors declare no competing financial interest.

\section{ACKNOWLEDGMENTS}

Support from Ministerio de Economía y Competitividad (CTQ2014-55583-R, CTQ2014-61914-EXP, CTQ2015-71955REDT) and Junta de Castilla y León (BU033-U16) is gratefully acknowledged. J.G.-R. thanks Ministerio de Educación, Cultura y Deporte for his FPU fellowship.

\section{REFERENCES}

(1) Kuwana, T.; Darlington, R. K.; Leedy, D. W. Anal. Chem. 1964, 36, 2023-2025.

(2) Heineman, W. R. J. Chem. Educ. 1983, 60, 305-308.

(3) Itoh, T.; McCreery, R. L. J. Am. Chem. Soc. 2002, 124, 10894 10902.

(4) Kaim, W.; Schwederski, B.; Dogan, A.; Fiedler, J.; Kuehl, C. J.; Stang, P. J. Inorg. Chem. 2002, 41, 4025-4028.

(5) Takashima, T.; Hashimoto, K.; Nakamura, R. J. Am. Chem. Soc. 2012, 134, 1519-1527.

(6) Pigani, L.; Heras, A.; Colina, Á.; Seeber, R.; López-Palacios, J. Electrochem. Commun. 2004, 6, 1192-1198.

(7) Baran, D.; Balan, A.; Celebi, S.; Meana Esteban, B.; Neugebauer, H.; Sariciftci, N. S.; Toppare, L. Chem. Mater. 2010, 22, 2978-2987.

(8) Kumar, R.; Pillai, R. G.; Pekas, N.; Wu, Y.; McCreery, R. L. J. Am. Chem. Soc. 2012, 134, 14869-14876.

(9) Richey, F. W.; Dyatkin, B.; Gogotsi, Y.; Elabd, Y. A. J. Am. Chem. Soc. 2013, 135, 12818-12826.

(10) Fernandez-Blanco, C.; Heras, A.; Ruiz, V.; Colina, A. RSC Adv. 2014, 4, 45168-45173.

(11) Ibañez, D.; Romero, E. C.; Heras, A.; Colina, A. Electrochim. Acta 2014, 129, 171-176.

(12) Lee, Y. F.; Kirchhoff, J. R. J. Am. Chem. Soc. 1994, 116, 3599-3600.

(13) Nagatani, H.; Suzuki, S.; Fermín, D. J.; Girault, H. H.; Nakatani, K. Anal. Bioanal. Chem. 2006, 386, 633-638.

(14) Izquierdo, D.; Martinez, A.; Heras, A.; Lopez-Palacios, J.; Ruiz, V.; Dryfe, R. A. W.; Colina, A. Anal. Chem. 2012, 84, 57235730 .
(15) Dahlman, C. J.; Tan, Y.; Marcus, M. A.; Milliron, D. J. J. Am. Chem. Soc. 2015, 137, 9160-9166.

(16) González-Diéguez, N.; Colina, A.; López-Palacios, J.; Heras, A. Anal. Chem. 2012, 84, 9146-9153.

(17) Syed, S. N.; Schulze, H.; Macdonald, D.; Crain, J.; Mount, A. R.; Bachmann, T. T. J. Am. Chem. Soc. 2013, 135, 5399-5407.

(18) Garoz-Ruiz, J.; Izquierdo, D.; Colina, A.; Palmero, S.; Heras, A. Anal. Bioanal. Chem. 2013, 405, 3593-3602.

(19) Reybier, K.; Nguyen, T. H. Y.; Ibrahim, H.; Perio, P.; Montrose, A.; Fabre, P.-L.; Nepveu, F. Bioelectrochemistry 2012, 88, $57-64$.

(20) Shi, Y.; Slaterbeck, A. F.; Seliskar, C. J.; Heineman, W. R. Anal. Chem. 1997, 69, 3679-3686.

(21) Pinyayev, T. S.; Seliskar, C. J.; Heineman, W. R. Anal. Chem. 2010, 82, 9743-9748.

(22) Asadpour-Zeynali, K.; Maryam Sajjadi, S.; Taherzadeh, F. Spectrochim. Acta Part A Mol. Biomol. Spectrosc. 2016, 153, 674680.

(23) Iijima, S. Nature 1991, 354, 56-58.

(24) Iijima, S.; Ichihashi, T. Nature 1993, 363, 603-605.

(25) Dresselhaus, M. S.; Dresselhaus, G.; Jorio, A. Annu. Rev. Mater. Res. 2004, 34, 247-278.

(26) Melchionna, M.; Marchesan, S.; Prato, M.; Fornasiero, P. Catal. Sci. Technol. 2015, 5, 3859-3875.

(27) De Volder, M. F. L.; Tawfick, S. H.; Baughman, R. H.; Hart, A. J. Science 2013, 339, 535-539.

(28) Schnorr, J. M.; Swager, T. M. Chem. Mater. 2011, 23, 646657.

(29) Fabbro, A.; Bosi, S.; Ballerini, L.; Prato, M. ACS Chem. Neurosci. 2012, 3, 611-618.

(30) Singhal, R.; Orynbayeva, Z.; Sundaram, R. V. K.; Niu, J. J.; Bhattacharyya, S.; Vitol, E. A.; Schrlau, M. G.; Papazoglou, E. S.; Friedman, G.; Gogotsi, Y. Nat. Nanotechnol. 2011, 6, 57-64.

(31) Hopley, E. L.; Salmasi, S.; Kalaskar, D. M.; Seifalian, A. M. Biotechnol. Adv. 2014, 32, 1000-1014.

(32) Hong, H.; Gao, T.; Cai, W. Nano Today 2009, 4, 252-261.

(33) Peng, G.; Tisch, U.; Haick, H. Nano Lett. 2009, 9, 1362-1368.

(34) Kruss, S.; Hilmer, A. J.; Zhang, J.; Reuel, N. F.; Mu, B.; Strano, M. S. Adv. Drug Deliv. Rev. 2013, 65, 1933-1950.

(35) Serafín, V.; Martínez-García, G.; Agüí, L.; Yáñez-Sedeño, P.; Pingarrón, J. M. Analyst 2014, 139, 4556-4563.

(36) Bianco, A.; Kostarelos, K.; Prato, M. Chem. Commun. 2011, 47, 10182-10188.

(37) Dumitrescu, I.; Unwin, P. R.; Macpherson, J. V. Chem. Commun. 2009, 6886-6901.

(38) Vashist, S. K.; Zheng, D.; Al-Rubeaan, K.; Luong, J. H. T.; Sheu, F.-S. Biotechnol. Adv. 2011, 29, 169-188.

(39) Mao, X.; Rutledge, G. C.; Hatton, T. A. Nano Today 2014, 9, 405-432.

(40) Bertoncello, P.; Edgeworth, J. P.; Macpherson, J. V; Unwin, P. R. J. Am. Chem. Soc. 2007, 129, 10982-10983.

(41) Garoz-Ruiz, J.; Palmero, S.; Ibañez, D.; Heras, A.; Colina, A. Electrochem. Commun. 2012, 25, 1-4.

(42) Garoz-Ruiz, J.; Ibañez, D.; Romero, E. C.; Ruiz, V.; Heras, A.; Colina, A. RSC Adv. 2016, 6, 31431-31439.

(43) Swamy, B. E. K.; Venton, B. J. Analyst 2007, 132, 876-884.

(44) Cheng, H.; Wang, X.; Wei, H. Anal. Chem. 2015, 87, 88898895.

(45) Liju, Y.; Yang, C. F.; Tuzhi, P.; Hangsheng, Y.; Cong, G.; Guoqing, L. Electroanalysis 1999, 11, 438-442.

(46) Cahill, P. S.; Walker, Q. D.; Finnegan, J. M.; Mickelson, G. E.; Travis, E. R.; Wightman, R. M. Anal. Chem. 1996, 68, 31803186.

(47) Ariansen, J. L.; Heien, M. L. A. V; Hermans, A.; Phillips, P. E. M.; Hernadi, I.; Bermudez, M. A.; Schultz, W.; Wightman, R. M. Front. Behav. Neurosci. 2012, 6, 1-10.

(48) Liu, Y.; Kim, H.; Franklin, R. R.; Bond, D. R. ChemPhysChem 2011, 12, 2235-2241. 
(49) Millo, D.; Harnisch, F.; Patil, S. A.; Ly, H. K.; Schröder, U.; Hildebrandt, P. Angew. Chemie Int. Ed. 2011, 50, 2625-2627.

(50) El-Said, W. A.; Kim, T.-H.; Chung, Y.-H.; Choi, J.-W. Biomaterials 2015, 40, 80-87.

(51) Van Dyke, D. A.; Cheng, H.-Y. Anal. Chem. 1988, 60, 12561260.

(52) Maizels, M.; Stegemiller, M.; Ross, S.; Slaterbeck, A.; Shi, Y.; Ridgway, T. H.; Heineman, W. R.; Seliskar, C. J.; Bryan, S. A. In Nuclear Site Remediation; Eller, P. G., Heineman, W. R., Eds.; American Chemical Society: Washington, DC, 2000; pp 364-378.

(53) Stegemiller, M. L.; Heineman, W. R.; Seliskar, C. J.; Ridgway, T. H.; Bryan, S. A.; Hubler, T.; Sell, R. L. Environ. Sci. Technol. 2003, 37, 123-130.

(54) Garoz-Ruiz, J.; Heras, A.; Palmero, S.; Colina, A. Anal. Chem. 2015, 87, 6233-6239.

(55) Ibañez, D.; Garoz-Ruiz, J.; Heras, A.; Colina, A. Anal. Chem. 2016, 88, 8210-8217.

(56) Deiss, F.; Sojic, N.; White, D. J.; Stoddart, P. R. Anal. Bioanal. Chem. 2010, 396, 53-71.
(57) Eggersdorfer, M.; Laudert, D.; Létinois, U.; McClymont, T.; Medlock, J.; Netscher, T.; Bonrath, W. Angew. Chemie Int. Ed. 2012 $51,12960-12990$.

(58) Pisoschi, A. M.; Pop, A.; Serban, A. I.; Fafaneata, C. Electrochim. Acta 2014, 121, 443-460.

(59) Kobayashi, H.; Akamine, H.; Okawa, Y.; Ohno, T.; Mizusawa, S. Electrochim. Acta 1991, 36, 1649-1651.

(60) Ye, B.-X.; Lin, L.; Wang, C.-H.; Jin, B.-H.; Liu, L.-F. J. Chinese Chem. Soc. 2002, 49, 415-419.

(61) DiVirgilio-Thomas, J. M.; Heineman, W. R.; Seliskar, C. J. Anal. Chem. 2000, 72, 3461-3467.

(62) Rueda, M.; Aldaz, A.; Sanchez-Burgos, F. Electrochim. Acta 1978, 23, 419-424.

(63) Berg, R. W. Appl. Spectrosc. Rev. 2015, 50, 193-239.

(64) Bielski, B. H. J.; Comstock, D. A.; Bowen, R. A. J. Am. Chem. Soc. 1971, 93, 5624-5629.

(65) In Official Methods of Analysis of AOAC International; Cunniff, P., Ed.; AOAC International: Gaithersburg, 1997; pp 16-17.

Insert Table of Contents artwork here

\section{Spectroelectrochemistry}

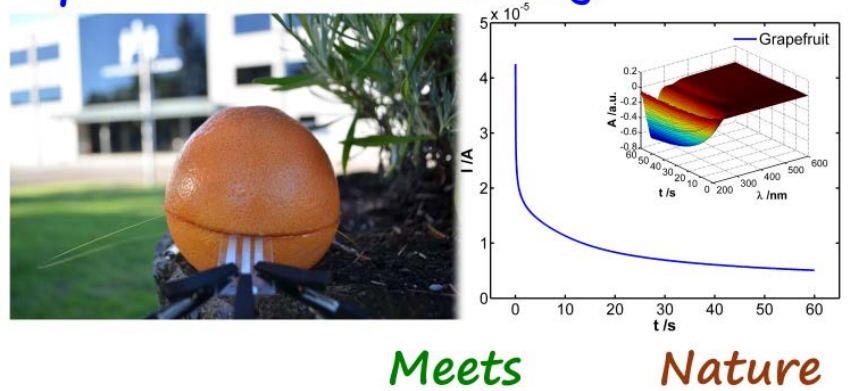

